\title{
Factores que influyen en la aceptación de dispositivos inteligentes insertables en el cuerpo
}

\section{Factors influencing the acceptance of intelligent devices inserted into the body}

\author{
Karina Carvajal $^{1 \dagger} \quad$ Alejandro Cataldo ${ }^{2 *} \quad$ María Amparo Mejía ${ }^{3}$ \\ Guiovanna Paola Sabogal-Alfaro ${ }^{4}$ \\ Recibido 25 de marzo de 2019, aceptado 26 de agosto de 2020 \\ Received: March 25, 2019 Accepted: August 26, 2020
}

\begin{abstract}
RESUMEN
Hasta hace poco, la posibilidad que los seres humanos portáramos dispositivos electrónicos en nuestro cuerpo era sólo planteado por la ciencia ficción. Esto ha cambiado con la aparición de los dispositivos insertables, aparatos electrónicos que las personas se introducen para asegurar confidencialidad en información o aumentar el control de acceso a recursos estratégicos. Dado que esta tecnología se encuentra aún en sus estados iniciales de adopción, existen muy pocos estudios que identifiquen los factores que influencian a las personas a aceptar/rechazar este tipo de aparatos.

Nosotros estudiamos un conjunto de nueve factores extraídos de una versión extendida de UTAUT y que podrían predecir la disposición a usar insertables. Los datos fueron recolectados mediante la aplicación de una encuesta a 183 estudiantes universitarios chilenos y fueron analizados usando un modelo de ecuaciones estructurales. Los resultados indican que cinco factores afectan la intención de usar insertables: hábito, motivación hedónica, influencia social, expectativa de esfuerzo y de rendimiento, explicando el 73,6\% de la varianza de la intención de uso de insertables. Factores relacionados al juicio ético de los respondientes no fueron significativos de la intención de uso. Estos dispositivos tienen un enorme potencial de innovación y económico que recién está dando los primeros pasos. En un futuro mediano, las personas podrían voluntariamente insertarse estos aparatos para mejorar sus funciones cognitivas y/o las empresas podrían pedir a sus empleados colocarse estos dispositivos para seguridad y control. Esto obligará a los países a regular la utilización de insertables, tal que su uso se dé en un marco legal que impida potenciales abusos de parte de las personas o las empresas en lo que sería cercano al mundo Orweliano. Consecuentemente, los resultados de este estudio son de interés tanto a la comunidad académica como profesional.
\end{abstract}

Palabras clave: Dispositivos insertables, aceptación TIC, UTAUT2.

\section{ABSTRACT}

Until recently, the possibility that we humans carry electronic devices in our bodies was only posed by science fiction. Today, this has changed with the emergence of insertable devices, electronic devices that people introduce into their bodies either to ensure confidentiality of information or to increase control

\footnotetext{
$1 \dagger$ Departamento Ingeniería Informática y Ciencias de la Computación. Universidad de Atacama. Av. Copayapu 485. Copiapó, Chile.

2 Escuela de Ingeniería Informática Empresarial. Universidad de Talca. Av. Lircay S/N, Talca, Chile. E-mail: acataldo@utalca.cl

3 Departamento de Humanidades. Universidad El Bosque. Calle 132 № 7 A-35 Casa X3. Bogotá, Colombia. E-mail: amparomejia@unbosque.edu.co

4 Facultad de Ingeniería. Universidad El Bosque. Edificio Fundadores Av. Carrera 9 No 131 A - 02. Bogotá - Colombia. E-mail: guiovannasabogal@unbosque.edu.co

* Autor de correspondencia: acataldo@utalca.cl
} 
of access to strategic resources. Since this technology is still in its initial stages of adoption, very few studies identify the factors that influence people to accept or reject this type of device. We studied a set of nine factors that could predict the intention to use insertable. The data were collected by applying a survey to 183 Chilean university students and analyzed using a structural equation model. The results show that five factors affect the intention to use insertable devices: habit, hedonic motivation, social influence, effort expectations, and performance expectations, explaining $73.6 \%$ of the variance. Factors related to the ethical judgment of respondents were not significant to the intended use. Insertable devices have an enormous potential for innovation and economic that is just taking the first steps. In the medium-term, people could voluntarily insert these devices to increase their cognitive functions and companies could force their employees to put on these devices for security and control. This will oblige countries to regulate the use of insertable devices, such that their use takes place within a legal framework that prevents potential abuses by individuals or companies, avoiding falling into something close to the Orwellian world. Consequently, the results of this study are of interest to both the academic and professional communities.

Keywords: Insertable devices, ICT acceptance, UTAUT2.

\section{INTRODUCCIÓN}

En el capítulo -toda tu historia- de la afamada serie inglesa-Blackmirror-, los protagonistas viven en una sociedad en la que es normal grabar todos lo que a las personas les ocurre a través de un chip de memoria que es introducido cuando nacen [1]. Con este dispositivo, las personas reproducen cualquier momento de su vida en cualquier smartTV disponibles en sus casas o en los taxis. En la película las personas pueden compartir momentos con sus amigos y tener momentos íntimos de introspección. Pero también las empresas usan estos recuerdos grabados para inspeccionar potenciales candidatos a contratar y los sistemas de seguridad nacionales los utilizan para descubrir potenciales intenciones terroristas.

Aunque esta historia que es sacada de una realidad Orweliana pareciera ser demasiada fantasiosa, tienen elementos de realidad que son más cercanos que fantasiosos. En efecto, dado que los dispositivos digitales cada vez son más potentes y pequeños, la posibilidad de introducir dispositivos inteligentes que incrementen alguna capacidad cognitiva o ayuden a mejorar los accesos a sitios o recursos delicados, es una realidad. Por ejemplo, el 2004, en México, se usó la tecnología RFID en una oficina de abogados para permitir el acceso a sitios seguros [2]. En ese mismo año, Verichip comercializó el primer chip aprobado por la FDA (Food and Drugs Administration) para uso en humanos. Para el 2007 se reporta que personas se han auto implantado RFIDs que se consiguen de manera comercial [2].
Los dispositivos móviles han pasado de ser portables (que pueden ser transportados con cierto esfuerzo por una persona), vestibles o wearables, a ser dispositivos -insertables-, es decir, artefactos digitales que se introducen dentro del cuerpo humano, tales como chip como subcutáneos, con el objeto de mejorar capacidades innatas o aumentar los niveles de seguridad [3]. Algunos ejemplos de aplicaciones en áreas diferentes a la salud son: compartir datos de contacto, desbloquear teléfonos, puertas y carros, ejecutar aplicaciones [4].

Los dispositivos insertables, que pueden parecer tecnología del futuro, son una realidad desde que Kevin Warwick en 1998 por primera vez se insertó bajo su piel un chip RFID (Radio Frecuency IDentification), para ingresar y prender las luces de su oficina, y lograr que su computador lo saludara [2]. Actualmente se encuentran tres tipos de insertables tecnológicos de uso común: chips RFID pasivo (identificación por radiofrecuencia), chips NFC (Near Field Communication), e imanes de neodimio [4].

A pesar de este gran surgimiento, la investigación sobre los factores que afectan la adopción de estos dispositivos por parte de los individuos es muy escasos aún. La necesidad de un estudio sobre este tema adquiere importancia, considerando que estas tecnologías se han incrementado en los últimos años y abrirán nuevas formas de modelos de negocios. De hecho, esta evolución tecnológica, les impone grandes retos éticos, morales y económicos a los 
sectores empresariales, a los estados nacionales y a la sociedad civil.

Para colaborar en cerrar esta brecha, este artículo presenta los resultados de un estudio que tiene como propósito identificar los factores que afectarían la decisión de adoptar dispositivos insertables entre un grupo de estudiantes de pregrado de una universidad chilena. Para el estudio se utilizó un modelo de nueve hipótesis analizadas mediante ecuaciones estructurales. La muestra correspondió a datos obtenidos de 152 encuestas de un total de 183 aplicadas a estudiantes universitarios chilenos.

La siguiente parte de este artículo está organizada así: la segunda sección resume el marco teórico y las hipótesis que guiaron esta investigación. La tercera, describe el método usado. La cuarta sección, presenta los resultados obtenidos mediante el uso de PLS-SEM (Partial Least Squares Structural Equation Modeling). Posteriormente se discuten los resultados obtenidos y por último se resumen las principales conclusiones de este estudio.

Trabajos relacionados y proposición de hipótesis El desarrollo de la tecnología y uso de los insertables se ha incrementado en los últimos años. Al mismo tiempo, el estigma social asociado con dichos dispositivos se ha ido reduciendo con el paso del tiempo. La familiarización de las personas con objetos insertables no tecnológicos en el cuerpo, como anticonceptivos o piercings, ha contribuido al surgimiento y aceptación del uso de dispositivos insertables tecnológicos [6]. Junto con este crecimiento, han surgido tendencias en el desarrollo de aplicaciones para el consumidor. La rápida evolución de TIC ha modificado radicalmente la forma en que los consumidores usan información [7]. Lo anterior puede ser indicativo de las influencias en el diseño y desarrollo de productos, así como de las influencias sociales entre los consumidores.

Aunque el mercado y los individuos ya han empezado a usar este tipo de tecnologías, aún existe poca investigación sobre el tema. De acuerdo con Michael [8] y Michael [9], entre 2003 y 2017 se han conducido algunos estudios en Estados Unidos, Australia, Alemania e Inglaterra acerca de la percepción de uso de estos implantes en humanos para usos no médicos. Los autores concluyen que, aunque la percepción positiva de uso en estos países ha aumentado, aún los resultados no son de todo concluyentes y dependen del área de aplicación. Perakslis and Wolk [10] realizaron una encuesta sobre la percepción de uso de estos dispositivos a estudiantes de dos universidades en Estados Unidos con un promedio de edad de 21 años. Los resultados reportan que la percepción de uso depende del área de aplicación, encontrándose que en aquella en donde habría una mayor aceptación para el uso de estos implantes (43\%) es la relacionada con aplicaciones que puedan ser utilizadas para salvar la vida o brindar seguridad para la familia. Johnston, et al. [11] realizaron un estudio en Australia, indagando sobre el conocimiento de los implantes RFID y su potencial uso. Los resultados mostraron que los australianos no están muy familiarizados con esta tecnología, sin embargo, valorarían su potencial uso en aplicaciones referentes a la seguridad nacional. Cuando se estudió la percepción de uso de estos dispositivos en propietarios de pequeñas empresas en Australia, Estados Unidos, Reino Unido e India, se encontró que un alto componente de la decisión de uso estaba influenciada por las implicaciones sociales y culturales de cada país [12]. En un estudio a estudiantes nacidos entre 1981 y 2000 , denominados millennials, se encontró que tienen una mayor tendencia al uso de estos dispositivos que sus generaciones predecesoras [13].

Acerca de las barreras asociadas a los implantes de microchips en el cuerpo, Perakslis, et al. [14] investigaron las barreras percibidas de implantar chips RFID para el control de acceso entre dueños de pequeñas empresas de Australia, India, Inglaterra y Estados Unidos. Los autores concluyeron que, aunque los participantes perciben el uso de tecnología con gran potencial, existe entre ellos barreras sociales y culturales que dificultan su uso. Entre estas barreras se encuentran: percepción de inseguridad en la tecnología, factores éticos (aunque estos cambian de acuerdo al país) y los posibles riesgos en la salud que podría traer el uso de estos implantes.

Uno de los trabajos iniciales que busca determinar los factores que afectan la disposición a usar este tipo de dispositivos es el realizado por [15], quienes analizaron un modelo causal para determinar la intención de usar implantes tecnológicos. Los autores usaron una versión modificada del modelo de aceptación tecnológica (TAM) para identificar los predictores de intención de uso. Además, incluyeron 
factores afectivos y normativos como predictores de la intención de uso. En su estudio, PelegrínBorondo, et al. [15] encontraron que percepción de facilidad de uso y de utilidad; emociones positivas y negativas hacia estas tecnologías; y normas subjetivas son predictores de intención de uso. Su estudio incluyó 600 personas mayores de 16 años residentes en España.

El trabajo de Pelegrín-Borondo, et al. [15] es un importante avance en la investigación de los dispositivos insertables, sin embargo, el uso de TAM como marco de referencia para construir el modelo causal le impone limitaciones, ya que la investigación de Pelegrín-Borondo, et al. [15] tácitamente asume que los individuos actuarán como consumidores de estas tecnologías, ya que se asume que la aceptación es un paso inicial para la adopción final [16]. Aunque TAM es un modelo ampliamente estudiado [17], no fue concebido como una teoría desde la perspectiva del consumidor. Por lo tanto, no incluye predictores que han sido identificados en la literatura como relevantes en estos contextos, por ejemplo, motivación hedónica o hábito [18]. En ese sentido, para entender la aceptación de los insertables sería recomendable usar como marco de referencias modelos que asumen una perspectiva de consumidor.

En la línea anterior, un modelo ampliamente aceptado y más actualizado que TAM es UTAUT2 [18]. UTAUT2 incorpora constructos propios de la aceptación tecnológica desde la perspectiva de los consumidores. UTAUT2 es una versión actualizada del modelo unificado de adopción y uso de tecnología (UTAUT) desarrollado por Venkatesh, et al. [19], que es un modelo construido a partir de la integración de ocho teorías previas de la aceptación tecnológica, incluida TAM. Mientras que UTAUT explica el $52 \%$ de la varianza de la intención de uso de una tecnología, UTAUT2 logra explicar el 74\% de la varianza [18].

En este estudio proponemos el uso de UTAUT2 como marco de trabajo por tres razones. Primero, UTAUT y UTAUT2 son los modelos más validados entre los investigadores de la adopción de una tecnología [18, 20, 22, 34]. UTAUT2 tiene un alto poder predictor del uso de tecnologías de consumo (74\%) [18]. Por último, es un modelo que busca explicar la aceptación de tecnologías de consumo, como lo son los insertables, por lo que incluye factores específicos que influencian a los potenciales consumidores a usar estas tecnologías.

\section{Proposición de hipótesis}

Basados en el modelo UTAUT2 se propusieron seis hipótesis que se describen a continuación.

Expectativa de rendimiento se define como el grado en el cual un individuo cree que usar un sistema le será útil en su vida [20]. Diversos estudios consistentemente demuestran que la expectativa de rendimiento es un predictor de la intención a usar una tecnología. Davis [21], usando el término -percepción de utilidad- encontró que los individuos decidían aceptar usar una nueva tecnología por la utilidad esperada que ellos creían que tendría un nuevo sistema. Venkatesh, et al. [19] también encontraron que los individuos usaban nuevas tecnologías esperando que éstas le fueran útiles. Más tarde, Venkatesh, et al. [18] encontraron que existía una relación positiva entre expectativa de rendimiento e intención de uso de una nueva tecnología entre consumidores. Ramírez, et al. [22] encontraron que la expectativa de rendimiento fue el segundo factor más importante para predecir el uso de Internet móvil entre estudiantes universitarios brasileños. Kohnke, et al. [20] encontraron que la expectativa de rendimiento es el principal predictor de la aceptación de sistemas de telemedicina por parte de pacientes en las clínicas. Por lo tanto, esto nos lleva a plantear la primera hipótesis de esta investigación:

\section{H1: La expectativa de rendimiento (PE) de los insertables influenciará positivamente la intención de usarlos (BI).}

Por otra parte, la Expectativa de esfuerzo está definida como el grado de facilidad asociado con el uso del sistema [19]. Ésta influencia directamente la intención de uso y tiene un impacto mayor para las mujeres, trabajadores de mayor edad y aquellos con experiencia limitada [19]. Diversos estudios consistentemente demuestran que la expectativa de esfuerzo es un predictor de la intención para usar una tecnología [16, 20]. Kohnke, et al. [20] confirma que la expectativa de esfuerzo es un importante predictor de la aceptación de sistemas de telemedicina por parte de pacientes en las clínicas. Farooq, et al. [23] confirman que la expectativa de 
esfuerzo tiene un rol significativo en la adopción de sistemas de captura de conferencias (LCS, Lecture Capture System). Nair, et al. [24] concluyeron que la expectativa de esfuerzo tiene una influencia significativa en la intención de uso de tecnologías de videograbación. Esto nos lleva a plantear la segunda hipótesis de esta investigación:

\section{H2: La expectativa de esfuerzo (EE) de los insertables influenciará positivamente la intención de usarlos (BI).}

La Influencia social es definida por Venkatesh, et al. [19] como la influencia que tienen las personas que son importantes para un sujeto en la decisión de utilizar un sistema nuevo. Algunos estudios demuestran que la influencia social tiene una relación positiva en la intención de uso de un dispositivo. Gao, et al. [25] encontraron que la influencia social estaba fuertemente relacionada con la decisión de adoptar tecnologías -vestibles- en el contexto de la salud. Más tarde, Pelegrín-Borondo, et al. [15] muestran que las normas subjetivas (uno de los componentes de la Influencia social) impactan de manera favorable la intención de uso de los dispositivos implantables. Recientemente, Piçarra and Giger [26] demostraron que este factor estaba asociado fuertemente con la intención de usar y trabajar con robots sociales. Además, concluyeron que la influencia social proveniente de los miembros reconocidos en una organización podría ser utilizada por los gerentes de las compañías para que sus empleados estén dispuestos a usarlos. De acuerdo con lo anterior se plantea la tercera hipótesis.

\section{H3: La influencia social favorable al uso de insertables (SI) influenciará positivamente la intención de usarlos (BI).}

La Motivación hedónica es definida como el placer o percepción de disfrute derivado del uso de una tecnología [18]. Se ha demostrado que ésta desempeña un papel importante en la determinación de la aceptación y uso de la tecnología. Gao, et al. [25] investigaron los factores asociados con la intención de uso de dispositivos -vestibles- en salud, encontrando que la motivación hedónica es un factor que impacta positivamente la percepción de uso de estos dispositivos, especialmente aquellos relacionados con el estado físico. Murata, et al. [27] hicieron estudios en jóvenes japoneses sobre la intención de uso y aceptación de tecnología ciborg. Ellos encontraron que la motivación hedónica es un factor que incide en la decisión de uso de estos dispositivos. De acuerdo con lo anterior se puede establecer que la motivación hedónica es un predictor de la intención de uso de los consumidores de tecnología, conduciendo a plantear la cuarta hipótesis:

\section{H4: La motivación hedónica (HM) influenciará} positivamente la intención de usar los dispositivos insertables (BI).

Los Hábitos favorables al uso de una tecnología es el grado en que las personas tienden a realizar conductas de forma automática debido al aprendizaje [18]. Diversos estudios demuestran que el hábito es un predictor de la intención a usar una tecnología. Nair, et al. [24] encontraron soporte para concluir el efecto significativo del hábito de los estudiantes sobre sus intenciones de uso y uso hacia tecnología de videograbación. Ramírez, et al. [22] encontraron que el hábito fue el factor más importante para predecir el uso de Internet móvil entre estudiantes universitarios brasileños. Por lo tanto, esto nos lleva a plantear la quinta hipótesis de esta investigación:

\section{H5: Hábitos favorables al uso de insertables (HT)} influenciará positivamente la intención de usarlos (BI).

La Relación precio/valor es la correspondencia entre los beneficios percibidos por un usuario al usar una aplicación/dispositivo y el costo monetario del mismo [18]. Alwahaishi and Snásel [16] encontraron que la apreciación de valor es un predictor significativo de la aceptación de tecnologías móviles entre consumidores. Ramírez, et al. [22] concluyen que la relación precio/valor es uno de los mayores determinantes para usar Internet Móvil. Para los dispositivos -vestibles-, Yang, et al. [28] concluyen que la relación precio/valor tiene una alta influencia tanto en los actuales usuarios como en potenciales. En un estudio realizado por Nawangsari, et al. [29] respecto a la aceptación de los consumidores a utilizar aplicaciones móviles, se encontró que la relación precio/valor influía en la intención de uso de dichas aplicaciones, demostrando además que esta relación es mayor en las mujeres. De acuerdo con lo anterior se plantea la sexta hipótesis: 
H6: Una mayor relación precio/valor de los dispositivos insertables (PV) influenciará positivamente la intención de usarlos (BI).

El juicio ético definido por Nguyen y Biderman [38] es un proceso cognitivo mediante el cual un individuo juzga qué acción es moralmente correcta. La relación que existe entre la intención que tienen las personas de usar dispositivos tecnológicos y lo que ellas consideran como éticamente correcto, ha sido poco estudiada. Puesto que el juicio ético es un constructo complejo, Reidenbach y Robin [39] Reidenbach, R.E. y Robin [40] afirman que su análisis es más efectivo a partir de un enfoque multidimensional. Los autores proponen tres dimensiones de la siguiente manera: La primera dimensión es equidad moral (equitativo y justo), referida a las creencias acerca de las conductas humanas correctas e incorrectas. La segunda es una dimensión relativa a los lineamientos y parámetros socioculturales que ayudan a definir las creencias éticas del individuo (aceptabilidad cultural y tradicional). La tercera, la dimensión contractual está relacionada con contratos, deberes, reglas existentes entre las organizaciones y la sociedad (legalidad, promesas no expresadas, contratos no escritos). Respecto al juicio ético en la intención de uso de tecnología, Wintersberger et al. [41] concluyeron que la equidad moral influye positivamente en la percepción de uso de tecnologías emergentes vehiculares. Por otra parte, en un estudio realizado por Peregrin-Borondo et al. [42] acerca de la influencia del juicio ético en la decisión de convertirse en un cyborg, con una muestra de 1.563 estudiantes universitarios en siete países, encontraron que esta variable podría afectar dicha decisión en casi un $50 \%$. Basado en lo anterior, postulamos las siguientes hipótesis (concretado en tres sub-hipótesis):

H7. El juicio ético influencia la intención de uso de los dispositivos insertables.

- H7.1: La equidad moral influencia positivamente la intención de uso de los dispositivos insertables.

- H7.2: El relativismo influencia positivamente la intención de uso de los dispositivos insertables.

- H7.3: El contractualismo influencia positivamente la intención de uso de los dispositivos insertables.

La Figura 1 muestra el modelo de hipótesis finalmente probado en este estudio.

\section{METODOLOGÍA}

Los datos fueron recolectados usando una encuesta a estudiantes universitarios de carreras de ingeniería y geología de la Universidad de Atacama, Chile. La recolección de datos consistió en un proceso de tres etapas. La primera fase fue la elaboración del cuestionario y su prueba a través de un pre-test aplicado a diez estudiantes de pregrado para eliminar

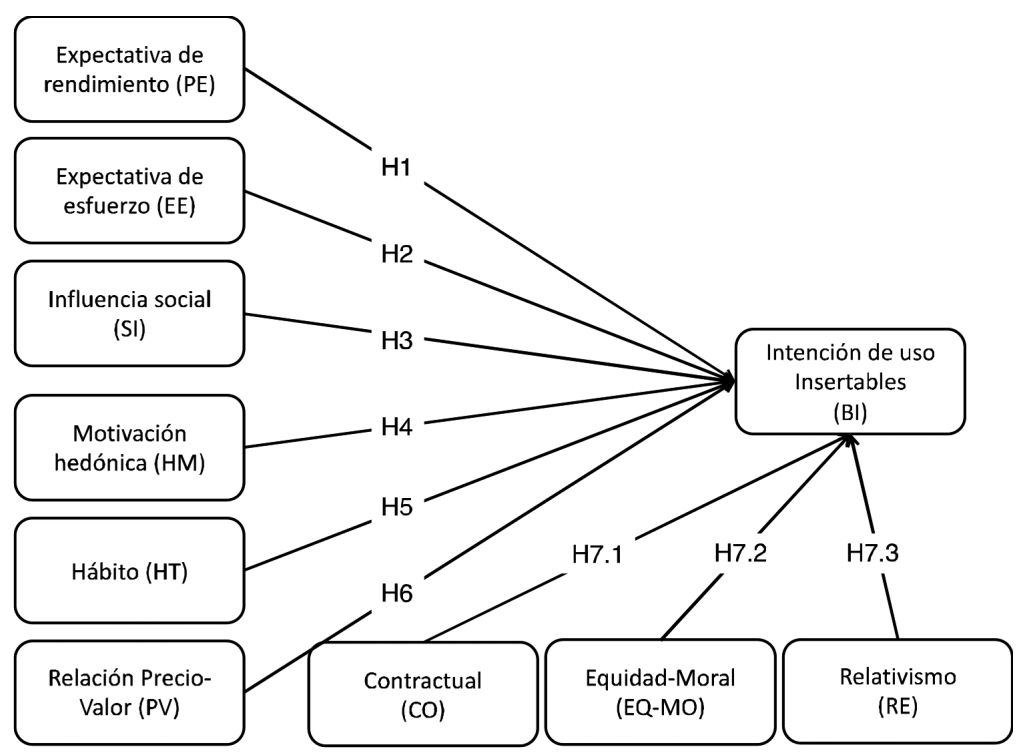

Figura 1. Modelo de hipótesis probado en esta investigación. 
errores de redacción y entendimiento. Las respuestas al pre-test no fueron incluidas en la base de datos final. Los cambios realizados después del pre-test fueron menores y principalmente de redacción de algunas preguntas. La segunda etapa consistió en la aplicación del cuestionario mediante una encuesta auto-administrada.

Dado que los dispositivos insertables son artefactos aún muy nuevos para la gran mayoría de los encuestados, se les pidió que, antes de contestar las preguntas, leyeran un texto introductorio (monografía) sobre qué son estos dispositivos y cómo se usan (esto puede ser solicitado a los autores). Se optó por usar dicho texto, para disminuir la variabilidad en las respuestas, dadas las diferencias en los énfasis que un presentador puede o no hacer, involuntariamente, en los distintos grupos. La última etapa fue el procesamiento de los datos. Para descubrir patrones de respuestas falsas, se agregaron dos preguntas especiales: una -pregunta trampa- y otra con la escala de respuesta invertida, esto permitió comprobar que los estudiantes realmente estaban respondiendo conscientemente. Todos los casos en los que estas preguntas fueron respondidas equivocadamente fueron descartados de la base de datos.

El cuestionario usado estuvo dividido en tres secciones (el cuestionario completo puede ser solicitado a los autores). Las preguntas de la primera sección fueron preguntas de tipo demográfico como: edad, carrera, sexo, entre otras. Otra sección tenía como propósito medir los siete constructos de UTAUT [18], dado que estos ítems están originalmente en inglés, se tradujeron al español y se compararon con las preguntas redactadas por [30] para su comprobación. Dichos constructos se midieron usando ítems de estudios ampliamente validados en la literatura para asegurar la validez de contenido [18, 19, 31]. La Expectativa de rendimiento (PE), Expectativa de esfuerzo (EE) y Hábito (HT) fueron medidos usando cuatro ítems. La Influencia Social (SI), Motivación Hedónica (HM), Relación Precio/Valor (PV) e Intención de uso (BI) fueron medidos usando tres ítems. Todos estos ítems fueron medidos empleando una escala Likert de cinco puntos que variaron desde -Totalmente en desacuerdo- a -Totalmente de acuerdo-. La otra sección tenía como objetivo indagar acerca de la influencia del juicio ético en la percepción de uso de estos dispositivos, utilizando una escala multidimensional de ocho elementos, basados en [39]. Cada uno de ellos fue medido presentando un escenario hipotético y utilizando una escala Likert de 7 puntos. La escala multidimensional ética de [40] utilizada para analizar el juicio ético de un sujeto, ha pasado por un proceso de depuración de cuatro etapas por sus autores, obteniendo un grado de fiabilidad con coeficiente entre 0,71 y 0,92 con una fiabilidad promedio de 0,8 . Finalmente, el total de ítems usados para esta investigación fue de 32 preguntas.

La encuesta fue tomada a 183 estudiantes de las carreras de ingeniería y geología de una universidad chilena. Diecinueve casos fueron eliminados por tener respuestas incompletas. De las 164 encuestas restantes, 12 de ellas respondieron mal la pregunta trampa o la respuesta invertida, por lo tanto, también fueron eliminadas. Finalmente, 152 encuestas fueron procesadas.

\section{RESULTADOS}

El modelo propuesto y las correspondientes hipótesis fueron probados usando PLS-SEM. El software utilizado fue SmartPLS 3.2.8. El análisis con PLS comenzó examinando el modelo de medición para evaluar la confiabilidad y validez de los constructos y los ítems. Posteriormente se probó el modelo estructural y sus hipótesis.

\section{Evaluación del modelo de medida}

La Tabla 1 y Tabla 2 presentan los resultados del modelo de medida, incluyendo información sobre la confiabilidad, validez y cargas del modelo de medición. Para la evaluación del modelo de medida, usamos las recomendaciones establecidas por Hair Jr, et al. [32]. La mayoría de los ítems fueron validados en Venkatesh, et al. [18] y [30]. Sin embargo, para este estudio los ítems fueron revalidados en términos de confiabilidad y validez.

Los patrones de carga y carga cruzada apoyaron la consistencia interna y validez discriminante. La confiabilidad de los indicadores fue evaluada analizando sus cargas. Como se puede apreciar en la Tabla 1, todas las cargas de los ítems respecto a su propio constructo, menos una de ellas, superan el criterio de ser mayor a 0,7 [32]. Un ítem de Hábito tuvo una carga menor a la esperada (HT2). Dado que su carga esta por sobre 0,6 y siguiendo 
Tabla 1. Cargas del modelo de medición.

\begin{tabular}{|c|c|c|c|c|c|c|c|c|c|c|}
\hline & BI & PE & EE & SI & HM & HT & PV & $\mathrm{CO}$ & EQ-MO & RE \\
\hline BI1 & 0,928 & & & & & & & & & \\
\hline BI2 & 0,963 & & & & & & & & & \\
\hline BI3 & 0,961 & & & & & & & & & \\
\hline PE1 & & 0,847 & & & & & & & & \\
\hline PE2 & & 0,885 & & & & & & & & \\
\hline PE3 & & 0,856 & & & & & & & & \\
\hline PE4 & & 0,817 & & & & & & & & \\
\hline EE1 & & & 0,882 & & & & & & & \\
\hline EE2 & & & 0,899 & & & & & & & \\
\hline EE3 & & & 0,856 & & & & & & & \\
\hline EE4 & & & 0,926 & & & & & & & \\
\hline SI1 & & & & 0,904 & & & & & & \\
\hline SI2 & & & & 0,899 & & & & & & \\
\hline SI3 & & & & 0,932 & & & & & & \\
\hline HM1 & & & & & 0,949 & & & & & \\
\hline HM2 & & & & & 0,931 & & & & & \\
\hline HM3 & & & & & 0,944 & & & & & \\
\hline HT1 & & & & & & 0,861 & & & & \\
\hline HT2 & & & & & & 0,671 & & & & \\
\hline HT3 & & & & & & 0,894 & & & & \\
\hline HT4 & & & & & & 0,923 & & & & \\
\hline PV1 & & & & & & & 0,817 & & & \\
\hline PV2 & & & & & & & 0,904 & & & \\
\hline PV3 & & & & & & & 0,806 & & & \\
\hline ETH-AGREE & & & & & & & & 0,920 & & \\
\hline ETH-SOC & & & & & & & & 0,952 & & \\
\hline ETH-ACEPT & & & & & & & & & 0,818 & \\
\hline ETH-EQUI & & & & & & & & & 0,708 & \\
\hline ETH-JUST & & & & & & & & & 0,832 & \\
\hline ETH-MORAL & & & & & & & & & 0,863 & \\
\hline ETH-CULT & & & & & & & & & & 0,903 \\
\hline ETH-TRAD & & & & & & & & & & 0,903 \\
\hline
\end{tabular}

Tabla 2. Indicadores de criterios de calidad del modelo de medición: Cronbach's Alpha, Rho_A, Composite Reliability, AVE y Heterotrait-Monotrait Ratio (HTMT).

\begin{tabular}{|l|l|l|l|l|l|l|l|l|l|l|l|l|l|}
\cline { 5 - 15 } \multicolumn{1}{c|}{} & $\begin{array}{c}\text { Cron-bach's } \\
\text { Alpha }\end{array}$ & rho_A & CR & AVE & BI & CO & EE & EQ-MO & HM & HT & PE & PV & RE \\
\hline BI & 0,947 & 0,947 & 0,966 & 0,904 & & & & & & & & & \\
\hline PE & 0,861 & 0,899 & 0,934 & 0,876 & 0,376 & & & & & & & & \\
\hline EE & 0,913 & 0,919 & 0,939 & 0,794 & 0,533 & 0,300 & & & & & & & \\
\hline SI & 0,826 & 0,861 & 0,882 & 0,652 & 0,482 & 0,853 & 0,421 & & & & & & \\
\hline HM & 0,936 & 0,94 & 0,959 & 0,886 & 0,781 & 0,346 & 0,463 & 0,412 & & & & & \\
\hline HT & 0,863 & 0,911 & 0,906 & 0,710 & 0,868 & 0,439 & 0,448 & 0,507 & 0,856 & & & & \\
\hline PV & 0,874 & 0,882 & 0,913 & 0,725 & 0,727 & 0,487 & 0,451 & 0,471 & 0,715 & 0,741 & & & \\
\hline CO & 0,811 & 0,95 & 0,881 & 0,712 & 0,396 & 0,344 & 0,398 & 0,236 & 0,488 & 0,471 & 0,364 & & \\
\hline EQ-MO & 0,774 & 0,774 & 0,898 & 0,815 & 0,457 & 0,914 & 0,311 & 0,785 & 0,422 & 0,511 & 0,535 & 0,301 & \\
\hline RE & 0,899 & 0,917 & 0,937 & 0,831 & 0,713 & 0,388 & 0,389 & 0,507 & 0,619 & 0,742 & 0,675 & 0,397 & 0,539 \\
\hline
\end{tabular}


las recomendaciones de Hair y otros, se examinaron los efectos sobre la confiabilidad y validez si el ítem era removido para decidir si sacarlos o no del modelo. Se comprobó que la remoción de HT2 afectaba la validez discriminante, por lo que se decidió mantener el ítem.

La consistencia interna (confiabilidad) queda establecida usando dos criterios mostrados en la Tabla 2: Confiabilidad compuesta (CR) y Alfa de Cronbach. En todos los constructos, ambos indicadores superan el umbral mínimo establecido de 0,7 [33].

A continuación, se evaluó la validez convergente. Esta quedó establecida usando el criterio del AVE. Como se puede apreciar en la Tabla 2, todos los AVE son superiores a 0,5 , establecido como umbral mínimo [32, 33], indicando una validez convergente satisfactoria.

La validez discriminante fue evaluada usando el criterio de Heterotrait-Monotrait Ratio (HTMT). Comúnmente para determinar la validez discriminante los investigadores usan el criterio de las cargas cruzadas o el de Fornell-Larcker [33]. Sin embargo, recientes investigaciones, han encontrado que estos criterios para evaluar la validez discriminante (cargas cruzadas y Fornell-Larcker) tienen problemas para detectar fallas de validez, cuando dos constructos están altamente correlacionados, por lo que Hair $\mathrm{Jr}$, et al. [32] sugieren que HTMT es un mejor indicador de validez discriminante. Este criterio establece que el HTMT no debe superar el valor 1,0 y que un umbral superior recomendable es 0,9 . $\mathrm{La}$ Tabla 2 muestra que todos los indicadores, menos uno (ETH-RE $\rightarrow$ ETH-CO), se encuentran dentro del rango aceptable. Para corregir el problema de validez discriminante entre ETH-RE y ETH-CO, se aplicó el criterio de las correlaciones sugerido por Hair Jr, et al. [32]. Los autores sugieren revisar la tabla de correlaciones entre ítems cuando hay problemas de validez discriminante, de tal forma de identificar ítems del mismo constructo (variable latente) con baja correlación y/o de diferentes constructos con alta correlación. Este análisis llevó a concluir que los ítems de Relativismo (ETH-TRAD y ETH-CULT) presentaban una correlación relativa baja. Dado que el ítem ETH-CULT presentó la más alta correlación con el constructo $\mathrm{CO}$, entonces se decidió remover. Una vez realizado esto, todos los indicadores HTMT cumplieron el criterio exigidos para lograr la validez discriminante.

\section{Modelo estructural}

La evaluación del modelo estructural se realizó mediante bootstrapping (5.000 sub-muestras, $\mathrm{BCa}$ bootstrap, two-tailed y nivel de significancia de $5 \%$ ). La Tabla 3 revela que cuatro de las nueve hipótesis son aceptadas. El constructo más influyente en la disposición a usar dispositivos insertables es Hábito $(\beta=0,454)$, seguido por Motivación hedónica $(\beta=0,168)$, a continuación, Influencia social $(\beta=0,148)$, Expectativa de rendimiento $(\beta=0,135)$, por último, el constructo menos influyente es Expectativa de esfuerzo $(\beta=0,131)$. La tabla también muestra que el constructo Relación precio/ valor no tuvo relación con la variable dependiente.

Tabla 3. Resultados de la prueba de boootstraping para evaluar el modelo estructural (5.000 sub-muestras, BCa bootstrap, two-tailed y nivel de significancia de 5\%).

\begin{tabular}{|l|l|c|c|c|c|c|l|}
\hline Hipótesis & \multicolumn{1}{|c|}{ Relación } & $\begin{array}{c}\text { Original } \\
\text { Sample }\end{array}$ & $\begin{array}{c}\text { Sample } \\
\text { Mean }\end{array}$ & $\begin{array}{c}\text { Standard } \\
\text { Deviation }\end{array}$ & T-Statistics & P-Values & Conclusión \\
\hline H1 & PE $\rightarrow$ BI & 0,132 & 0,135 & 0,065 & 2,046 & 0,041 & Soportada \\
\hline H2 & EE $\rightarrow$ BI & 0,134 & 0,131 & 0,045 & 2,966 & 0,003 & Soportada \\
\hline H3 & SI $\rightarrow$ BI & 0,148 & 0,147 & 0,076 & 1,952 & 0,050 & Soportada \\
\hline H4 & HM $\rightarrow$ BI & 0,168 & 0,160 & 0,085 & 1,971 & 0,049 & Soportada \\
\hline H5 & HT $\rightarrow$ BI & 0,454 & 0,455 & 0,086 & 5,258 & 0,000 & Soportada \\
\hline H6 & PV $\rightarrow$ BI & $-0,037$ & $-0,032$ & 0,060 & 0,628 & 0,530 & No soportada \\
\hline H7a & CO $\rightarrow$ BI & 0,038 & 0,037 & 0,076 & 0,503 & 0,615 & No soportada \\
\hline H7b & EQ-MO $\rightarrow$ BI & $-0,048$ & $-0,053$ & 0,069 & 0,691 & 0,489 & No soportada \\
\hline H7c & RE $\rightarrow$ BI & 0,017 & 0,018 & 0,062 & 0,275 & 0,783 & No soportada \\
\hline
\end{tabular}


Asimismo, ninguno de los sub-constructo de juicio ético tuvo significancia con la intención de usar dispositivos insertables.

La Figura 2 resume los resultados del modelo estructural analizado. Como se puede apreciar Expectativa de esfuerzo, Expectativa de esfuerzo, Influencia social, Motivación hedónica y Hábito, explican el 73,6\% de la varianza de la intención de usar dispositivos insertables $\left(\mathrm{R}^{2}=0,736\right)$ que es un valor moderado a sustantivo.

\section{Calidad del modelo}

Para medir la calidad del modelo se evaluaron tres aspectos: el efecto de tamaño $\left(\mathrm{f}^{2}\right)$, la relevancia predictiva del modelo (ver Tabla 4) y el calce del modelo. El efecto de tamaño es usado para medir el impacto que un constructo tiene sobre la variable dependiente [32]. Observando la Tabla 4 se puede apreciar que Hábito tiene un gran efecto $\left(\mathrm{f}^{2}>0,35\right)$, Expectativa de rendimiento tienen un efecto medio $\left(0,02>f^{2}>0,15\right)$ y los constructos Expectativas de esfuerzo, Motivación hedónica e Influencia social tienen un efecto pequeño $\left(\mathrm{f}^{2}>0,02\right)$. Los constructos Relación Precio/Valor y los de Juicio ético no tienen efecto sobre la Intención de uso.
Tabla 4. Efecto de tamaño e indicador de relevancia predictiva del modelo.

\begin{tabular}{|l|c|c|l|}
\cline { 2 - 4 } \multicolumn{1}{c|}{} & \multicolumn{1}{c|}{$\mathbf{f}^{\mathbf{2}}$} & \multicolumn{1}{c|}{$\mathbf{Q}^{\mathbf{2}}$} & \multicolumn{1}{c|}{ Conclusión } \\
\hline BI & & 0,581 & Relevancia predictiva \\
\hline PE & 0,030 & & Medio \\
\hline EE & 0,049 & & Pequeño \\
\hline SI & 0,037 & & Pequeño \\
\hline HM & 0,036 & & Pequeño \\
\hline HT & 0,222 & & Grande \\
\hline PV & 0,004 & & Sin efecto \\
\hline ETH-CO & 0,002 & & Sin efecto \\
\hline ETH-MO & 0,003 & & Sin efecto \\
\hline ETH-RE & 0,001 & & Sin efecto \\
\hline
\end{tabular}

La medición de la relevancia predictiva del modelo fue establecida mediante el valor $Q^{2}$ [32]. Observando la Tabla 4 y considerando los $\mathrm{f}^{2}$ obtenidos, dado que $Q^{2}$ es mucho mayor que cero, entonces los resultados entregan claro soporte a la relevancia predictiva del modelo respecto a la Intención de uso de los dispositivos insertables. Por último, para medir el calce del modelo se utilizó el residuo cuadrático medio estandarizado (SRMR), que fue de $0,068(<0,08)$, concluyendo que existe un buen calce del modelo [32].

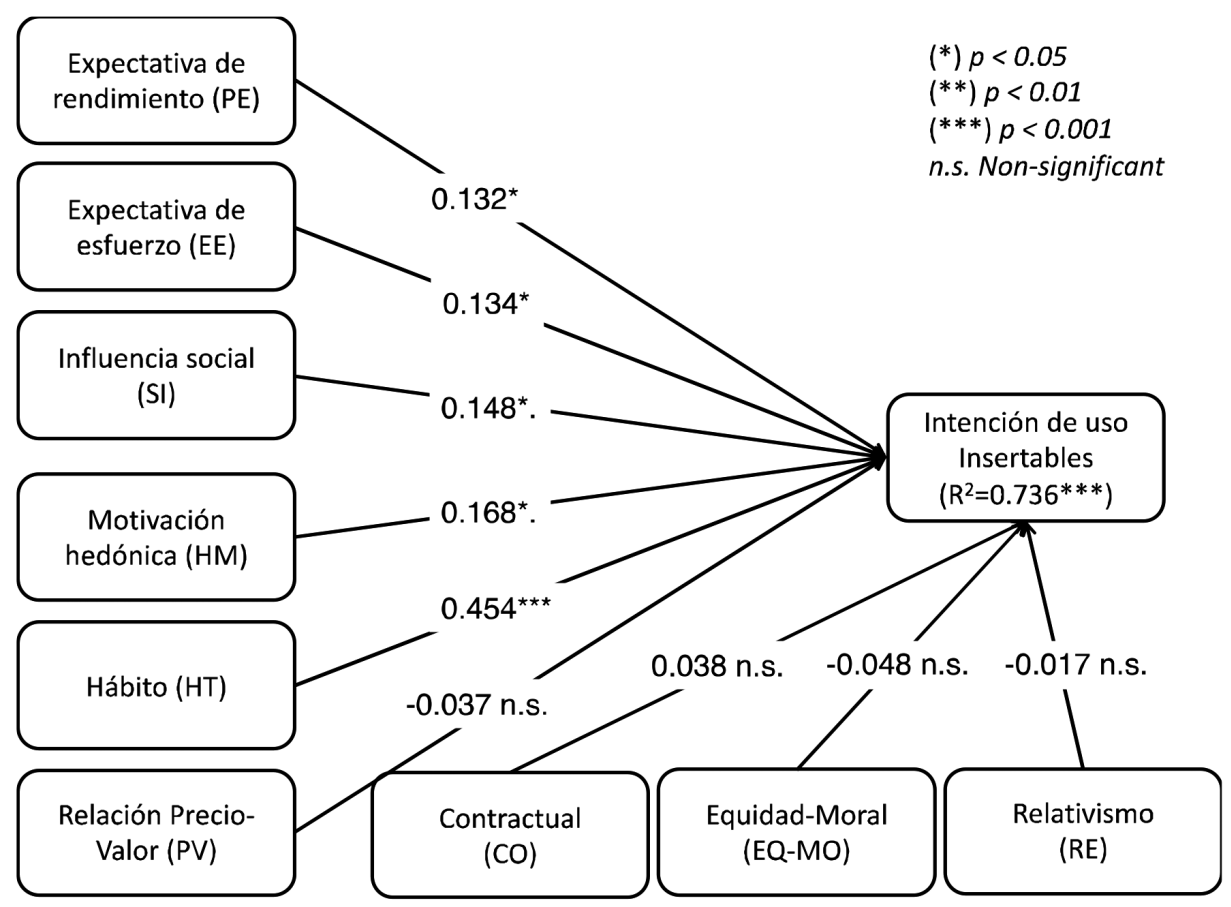

Figura 2. Modelo estructural resultante. 


\section{DISCUSIÓN}

Nuestros resultados confirman la validez de UTAUT en la intención de uso de insertables. Primero nuestros resultados confirman que la Expectativa de rendimiento tiene un efecto significativo sobre la intención de uso de los dispositivos insertables (H1). Estos resultados son consistentes con estudios previos $[18,20,22,34]$ que concluyeron que este constructo es uno de los mejores predictores de Intención de uso (en general). Sin embargo, su efecto es menor que el de Hábito, una mayor discusión y posible explicación es presentada al final de esta sección.

Asimismo, se confirma que Expectativa de esfuerzo tiene un efecto significativo sobre la intención de uso de los dispositivos insertables (H2), siendo este resultado también consistente con estudios previos [20]. Farooq, et al. [23] y Nair, et al. [24] concluyeron similarmente que la expectativa de esfuerzo tiene un rol significativo en la adopción de los distintos dispositivos. Similarmente, los resultados son consistentes con Barahona Vinasco and Calderón García [35] quienes encontraron que la percepción de facilidad de uso (equivalente en TAM a la expectativa de esfuerzo) es un predictor de la actitud hacia el uso de las tecnologías en la fuerza de ventas. Una explicación de este resultado es que las personas creen que la facilidad de usar los dispositivos insertables los motivaría a usarlos. Estudios han mostrado que la percepción de facilidad de uso (en el modelo TAM) o expectativa de esfuerzo (en el modelo UTAUT) es un predictor de la intención de uso de diversas tecnologías.

De la misma forma, se confirma que la Influencia social favorece el uso de insertables y que afecta positivamente la intención de utilizar estos dispositivos (H3). Estos resultados son consistentes con estudios previos $[15,1920,25,26]$, que encontraron que la influencia social es una variable relevante que influye en la intención de uso. Una posible explicación para el contexto particular latinoamericano es que culturalmente cualquier decisión de usar un elemento que modifique o afecte el cuerpo humano está altamente influenciada por las personas que son importantes para un sujeto como su familia o amigos.

También los resultados de este estudio confirman que la Motivación hedónica es un factor que influye positivamente en la intención de uso de los dispositivos insertables (H4), siendo consistentes con estudios previos [18, 25, 29]. Diversos autores han concluido que la motivación hedónica juega un papel determinante en la intención de uso, especialmente en jóvenes. Nuestro estudio confirma esta conclusión.

Con respecto a la hipótesis H5, se corrobora que los hábitos tienen un efecto significativo sobre la intención de uso de los dispositivos insertables. Estos resultados son consistentes con estudios previos [23, 24] que demuestran que el hábito es un predictor importante en la intención de usar una tecnología. Un resultado interesante es que este constructo fue el más relevante predictor de la intención de uso. Se puede especular que las personas más habituadas a usar diversas tecnologías también estarán más dispuestas a usar los dispositivos insertables. Cabe destacar que, según otros estudios hechos en Latinoamérica [30], el hábito es el primero o segundo constructo más influyente en la intención de uso de tecnologías asociadas a Internet móvil.

A diferencia de los hallazgos encontrados en estudios previos [16, 18, 22, 29], los resultados en este estudio no soportan la hipótesis H6, que afirma que una mejor relación precio/valor influencia la intención de usar dispositivos insertables. La relación precio/valor en esta investigación particular no es un factor que influencie la intención de uso de estos dispositivos. Estos resultados son consistentes con el estudio realizado por Ramírez-Correa [30], quien concluyó que este constructo no influenció la disposición a usar internet móvil en Chile. Una posible explicación a lo encontrado es que al ser los dispositivos insertables una tecnología no muy conocida en Latinoamérica no era fácil para los encuestados estimar la relación entre los costos directos (monetarios) y los beneficios obtenidos. No obstante, se requiere una mayor investigación para explorar la relación entre el constructo precio/valor y la disposición para usar dispositivos insertables.

Respecto a la influencia del juicio ético en la percepción del uso de los dispositivos insertables (H7: H7.1, H7.2, H7.3), los resultados indican que éste no influencia su aceptación. A diferencia de lo mostrado por [42], nuestro estudio no evidencia que los constructos de equidad moral, contractualismo y relativismo afecten la percepción de uso de estos 
dispositivos. Una causa de lo anterior podría deberse, en parte, al desconocimiento sobre este tipo de tecnologías en América Latina lo cual dificulta tomar una postura ética sobre ella. Por otro lado, es recomendable ampliar el número de escenarios en estudios futuros que permitan explorar con mayor profundidad las tres dimensiones del juicio ético como predictor o moderador del uso de dispositivos insertables.

Reflexionando sobre los resultados de esta investigación, hay un aspecto de éste que llaman la atención. Como fue mencionado en párrafos previos, aunque las Expectativas de rendimiento y de esfuerzo fueron significativas para la intención de uso, ambos constructos fueron los menos influyentes sobre la variable dependiente. Esto es un hallazgo diferente a la mayoría de los estudios sobre aceptación tecnológica que mayoritariamente muestran que estos dos constructos son los predictores más influyentes en la intención de uso de otras TIC [34]. En contraste, los constructos relacionados a la autoconfianza de los individuos (hábito y motivación hedónica) respecto a usar tecnologías, son los factores críticos que empujan a usar dispositivos insertables entre los jóvenes. En otras palabras, las actitudes (expectativas de esfuerzo y rendimiento) hacia los dispositivos insertables tienen un relativo bajo efecto en la decisión de usar estas tecnologías, mientras que la autoconfianza es mejor predictor, algo que es contrario a los resultados clásicos de la aceptación.

Una posible explicación de este resultado podría deberse al carácter muy novedoso que estas tecnologías tienen, en especial para la muestra estudiada. Desde la psicología, Kahneman [37] sostiene que cuando las personas deben tomar decisiones, intuitivamente comienzan con una primera aproximación (anchor), luego hacen ajustes según van recogiendo información (adjustment). En nuestro caso, los estudiantes participantes no conocían previamente estos tipos de dispositivos, por lo que no tenían más criterios de decisión que las heurísticas psicológicas descritas por Kahneman [37]. En consecuencia, hábito y motivación hedónica se transforman en los determinantes más relevantes para decidir usar dispositivos insertables, sobre otros relacionados a la actitud de usar tecnologías conocidas.

Otra explicación complementaria de este resultado es que, en las primeras etapas de uso de una nueva tecnología, los jóvenes tienen una mayor tendencia a buscar cosas nuevas. Esta tendencia aumenta la motivación hedónica, influyendo positivamente en la toma de decisiones para la adopción de una nueva TIC. Esto explicaría por qué la motivación hedónica fue el segundo constructo con mayor efecto sobre la intención de uso de insertables. Esta explicación es consistente con Jiménez, et al. [36] quienes encontraron que una mayor tendencia a disfrutar y experimentar con nuevas tecnologías aumentaba la confianza de los usuarios de e-mobile.

\section{CONCLUSIONES}

Al inicio de este estudio, se quiso enfatizar el valor potencial que los nuevos dispositivos insertables tienen como oportunidad de mercado en Latinoamérica. Por ello se determinaron las causales de aceptación de estas tecnologías emergentes entre los jóvenes chilenos universitarios. Los resultados muestran que cinco factores de los nueve propuestos afectan la intención a usar dispositivos insertables y juntos explican el 73,6\% de la varianza de intención de uso. Los predictores más relevantes fueron hábito y motivación hedónica, mostrando que factores relacionados con la heurística psicológica (autoconfianza según Kahneman [37]) son los que más pesan en la decisión de usar dispositivos insertables. Sorpresivamente, predictores como expectativas de rendimiento y de esfuerzo, que han sido largamente comprobados como factores claves de la intención de uso, tuvieron un nivel de influencia pequeño en la intención de uso, siendo incluso menos relevantes que la influencia social. Este es un resultado inusual porque diferentes estudios han indicado que expectativa de esfuerzo es uno de los que mejor califica como predictor de la intención de uso [34]. Claramente más investigación se necesita para esclarecer este resultado inesperado.

Las conclusiones de este estudio son de interés para investigadores y empresas. Los primeros pueden usar estos resultados para profundizar sobre los factores que influencian la adopción de tecnologías emergentes como los insertables, implantables y cyborgs, entre otros. La teoría de aceptación tecnológica ampliamente ha estudiado los factores que afectan la decisión de adopción de TIC, sin embargo, hay pocos estudios relacionados con tecnologías que aún no son ampliamente conocidas por la población como las mencionadas. Los resultados indicarían 
que cuando las tecnologías son muy emergentes, predictores bien conocidos bajan su influencia respecto a otros más relacionados con los sentimientos de autoeficacia (hábito y motivación hedónica) que poseen los individuos. Una mayor profundización de esta conclusión, así como su validación, debería ser tratada en trabajos futuros.

Este estudio puede ser relevante para las empresas innovadoras y/o interesadas en explorar nuevas oportunidades de mercado. Los dispositivos insertables forman parte de un grupo de nuevas tecnologías emergentes que están en las etapas muy iniciales de su ciclo de vida, y que prometen tener un gran campo de aplicación y una alta demanda en el mediano y largo plazo. Por lo tanto, en una primera fase de difusión, compañías que quieran desarrollar o distribuir dispositivos insertables, podrían enfocarse en construir campañas de marketing que promuevan estas tecnologías entre individuos habituados a usar TIC y con un alto grado de autoconfianza en su uso. En una segunda etapa, cuando estas tecnologías ya sean más conocidas, las empresas deberían volcar sus esfuerzos comerciales en los beneficios esperados, la imagen asociada a estas tecnologías y la facilidad de uso que los dispositivos insertables tienen.

Nuestro estudio tiene por lo menos dos limitaciones evidentes. Primero el tipo de muestra usada en este trabajo corresponde a un grupo muy particular de personas: jóvenes estudiantes universitarios de pregrado. Segundo, dado que la tecnología estudiada es emergente y desconocida para los participantes, el uso de una monografía como fuente de información sobre las características, ventajas y desventajas de los dispositivos insertables puede ser debatida. Estas limitaciones hacen que la generalización de los resultados del estudio se trate con precaución. Así mismo, estas limitaciones abren espacios para validar nuestras conclusiones o profundizarlas por parte de otros investigadores.

Finalmente, los dispositivos insertables son un grupo de tecnologías emergentes que se encuentran siendo usadas en nichos muy pequeños de usuarios o aun en espacios de experimentación. Sin embargo, son tecnologías que abren oportunidades de nuevos negocios y se proyecta que serán masificadas en un horizonte de no muy largo plazo. Por ello, es relevante que la academia y el mundo de las empresas pueden encontrar nichos de estudios y mercado nuevos. Latinoamérica tiene en ese sentido, una circunstancia especial para no quedarse atrás en la comercialización y uso de estas nuevas TIC. Al mismo tiempo, los gobiernos se enfrentarán a nuevos desafíos en términos de regular apropiadamente la utilización de dispositivos insertables, tal que su uso se de en un marco legal justo que impida potenciales abusos de parte de las empresas que traten de imponer su utilización a sus trabajadores.

\section{REFERENCIAS}

[1] B. Welsh. "The Entire History of You". In Black Mirror, Season 1, Chapter 3 ed. London: Netflix. 2011.

[2] M.N. Gasson, E. Kosta and D. M. Bowman "Human ICT implants: From invasive to pervasive (Human ICT implants: technical, legal and ethical considerations). Springer Science \& Business Media, pp. 1-10. 2012.

[3] K. Heffernan, F. Vetere, L. Britton, B. Semaan and T. Schiphorst. "Insertable Digital Devices: Voluntarily Under the Skin", in Conference on Designing Interactive Systems, Brisbane, Australia. Proceedings of the 2016 ACM Conference on Designing Interactive Systems, pp. 85-88: ACM. 2016.

[4] K. Heffernan, F. Vetere and S. Chang. "Insertables: I've got it under my skin". Interactions. Vol. $23 \mathrm{~N}^{\mathrm{o}}$ 1, pp. 52-56. 2015.

[5] IDC-Media-Center. Global Wearables Market Grows 7,7\% in 4Q17 and 10,3\% in 2017 as Apple Seizes the Leader Position. April 8, 2018.

[6] K. Heffernan, F. Vetere and S. Chang. "Towards insertables: Devices inside the human body". First Monday. Vol. $22 \mathrm{~N}^{\mathrm{o}} 3$, p. 1. 2017.

[7] B. Velázquez, M. Blasco and I. Gil-Saura. "ICT adoption in hotels and electronic word-ofmouth". Academia Revista Latinoamericana de Administración. Vol. 28 Nº 2, pp. 227-250. 2015.

[8] K. Michael. "Go "Get Chipped": A Brief Overview of Non-Medical Implants between 1997-2013 (Part 1)". IEEE Technology and Society Magazine. Vol. $36 \mathrm{~N}^{\circ}$ 3, pp. 6-9. 2017.

[9] K. Michael. "Go "Get Chipped": A Brief Overview of Non-Medical Implants between 1997-2013 (Part 2)", IEEE Technology and 
Society Magazine. Vol. 36 No 4, pp. 6-12. 2017.

[10] C. Perakslis and R. Wolk. "Social acceptance of RFID as a biometric security method". In Symposium on Technology and Society (ISTAS 2005), pp. 79-87. Los Angeles, CA, USA, IEEE. 2005.

[11] K. Johnston, K. Michael and M. Michael. "Consumer awareness in Australia on the prospect of humancentric RFID implants for personalized applications". In Sixth International Conference on, Toronto, Ontario, Canada. Piscataway, N.J.: IEEE. 2007.

[12] K. Michael, C. Perakslis and M. Michael. "Microchip implants for employees in the workplace: Findings from a multi-country survey of small business owners". Surveillance and/in Everyday Life: Monitoring Pasts. February 20-21, 2012.

[13] C. Perakslis and K. Michael. "Indian Millennials: Are microchip implants a more secure technology for identification and access control?". In IEEE Conference onTechnology and Society in Asia (T\&SA), pp. 1-9. IEEE. Singapore, Singapore. 2012.

[14] C. Perakslis, K. Michael, M. Michael and R. Gable. "Perceived barriers for implanting microchips in humans: A transnational study". In IEEE Conference on Norbert Wiener in the 21st Century (21CW), pp. 1-8. Boston, MA, USA, IEEE. 2014,

[15] J. Pelegrín-Borondo, E. Reinares-Lara and C. Olarte-Pascual. "Assessing the acceptance of technological implants (the cyborg): Evidences and challenges", Computers in Human Behavior. Vol. 70, pp. 104-112. 2017.

[16] S. Alwahaishi and V. Snásel. "Acceptance and use of information and communications technology: a UTAUT and flow based theoretical model". Journal of technology management \& innovation. Vol. $8 \mathrm{~N}^{\circ} 2$, pp. 61-73. 2013.

[17] A. J. Cataldo Cataldo and L. Zambra Alcayaga. "Usando Investigación-Acción para unir la práctica con la teoría en sistemas. Examinando cualitativamente la teoría de adopción de tecnología en una pyme". Innovar. Vol. 26 $\mathrm{N}^{\circ}$ 60, pp. 147-160. 2016.

[18] V. Venkatesh, J. Thong and X. Xu. "Consumer acceptance and use of information technology: extending the unified theory of acceptance and use of technology". MIS Quarterly. Vol. $36 \mathrm{~N}^{\circ}$ 1, p. 157.178. 2012.

[19] V. Venkatesh, M. Morris, G. Davis and F. Davis. "User acceptance of information technology: Toward a unified view". MIS quarterly. Vol. 27 No 3, pp. 425-478. 2003.

[20] A. Kohnke, M.Cole and R. Bush. "Incorporating UTAUT predictors for understanding home care patients' and clinician's acceptance of healthcare telemedicine equipment". Journal of technology management \& innovation. Vol. $9 \mathrm{~N}^{\circ}$ 2, pp. 29-41. 2014.

[21] F. Davis. "Perceived usefulness, perceived ease of use, and user acceptance of information technology", MIS quarterly. Vol. $13 \mathrm{~N}^{\circ} 3$, pp. 319-340. 1989.

[22] P. Ramírez, A. Mariano, J. Alfaro and M. Marión. "Aceptación de internet móvil en estudiantes universitarios brasileños: Un estudio empírico usando modelado de ecuaciones estructurales". Revista ESPACIOS. Vol. 36 N $^{\circ} 13$, pp. 10-18. 2015.

[23] M.S. Farooq et al. "Acceptance and use of lecture capture system (LCS) in executive business studies: Extending UTAUT2". Interactive Technology and Smart Education. Vol. $14 \mathrm{~N}^{\circ}$ 4, pp. 329348. 2017.

[24] P. Nair, F. Ali and L. Leong. "Factors affecting acceptance \& use of ReWIND: Validating the extended unified theory of acceptance and use of technology". Interactive Technology and Smart Education. Vol. 12 N$^{\circ} 3$, pp. 183201. 2015.

[25] Y. Gao, H. Li and Y. Luo. "An empirical study of wearable technology acceptance in healthcare". Industrial Management \& Data Systems. Vol. 115 No 9, pp. 1704-1723. 2015.

[26] N. Piçarra and J.-C. Giger. "Predicting intention to work with social robots at anticipation stage: Assessing the role of behavioral desire and anticipated emotions". Computers in Human Behavior. 2018.

[27] K. Murata, A. Adams, Y. Fukuta, Y. Orito, M. Arias-Oliva and J. Pelegrin-Borondo. "From a Science Fiction to the Reality. Cyborg Ethics in Japan", ORBIT Journal. Vol. $1 \mathrm{~N}^{\circ}$ 2, pp. 1-15. 2017.

[28] H. Yang, J. Yu, H. Zo, and M. Choi, "User acceptance of wearable devices: An extended 
perspective of perceived value. "Telematics and Informatics. Vol. $33 \mathrm{~N}^{\circ}$ 2, pp. 256-269. 2016.

[29] S. Nawangsari, E. P. Wibowo and R. Budiarto. "EMPIRICAL study on consumer acceptance of mobile applications in Jakarta Indonesia". In Informatics and Computing (ICIC), 2017 Second International Conference on, pp. 1-6. IEEE. 2017.

[30] P. Ramírez-Correa. "Uso de internet móvil en Chile: explorando los antecedentes de su aceptación a nivel individual”, Ingeniare. Revista chilena de ingeniería. Vol. $22 \mathrm{~N}^{\circ} 4$, pp. 560-566. 2014.

[31] I. Ajzen and M. Fishbein. "Understanding attitudes and predicting social behavior". NJ, USA: Prentice Hall. 1980.

[32] J. Hair Jr, G.T. Hult, C. Ringle and M. Sarstedt. "A primer on partial least squares structural equation modeling (PLS-SEM)". Los Angeles, CA: Sage Publications. 2016.

[33] J. Henseler, C.M. Ringle and R.R. Sinkovics. "The use of partial least squares path modeling in international marketing". In New challenges to international marketing: Emerald Group Publishing Limited, pp. 277319. 2009.

[34] M. Williams, N. Rana and Y. Dwivedi. "The unified theory of acceptance and use of technology (UTAUT): a literature review", Journal of Enterprise Information Management. Vol. $28 \mathrm{~N}^{\circ}$ 3, pp. 443-488. 2015.

[35] J.F. Barahona Vinasco and H. Calderón García. "La actitud del vendedor ante las innovaciones tecnológicas y su influencia en su desempeño en las ventas". Innovar. Vol. 27 No 66, pp. 29-39. 2017.
[36] N. Jimenez, S. San-Martin and J. Azuela. "Trust and satisfaction: the keys to client loyalty in mobile commerce", Academia Revista Latinoamericana de Administración. Vol. $29 \mathrm{~N}^{\circ}$ 4, pp. 486-510. 2016.

[37] D. Kahneman. "Thinking, fast and slow". New York, USA: Farrar, Straus and Giroux. 2011.

[38] N.T. Nguyen and M.D. Biderman. "Studying ethical judgments and behavioral intentions using structural equations: Evidence from the multidimensional ethics scale". Journal of Business Ethics. Vol. $83 \mathrm{~N}^{\circ}$ 4, pp. 627. 2008.

[39] R.E. Reidenbach and P.R. Donald. "Toward the development of a multidimensional scale for improving evaluations of business ethics". Journal of business ethics. Vol. 9 $\mathrm{N}^{\circ}$ 8, pp. 639-653. 1990.

[40] R.E. Reidenbach and P.R. Donald. "Toward the development of a multidimensional scale for improving evaluations of business ethics". Citation Classics from the Journal of Business Ethics. Springer, Dordrecht, pp. 45-67. 2013.

[41] P. Wintersberger, A.K. Prison, A. Riener and S. Hasirlioglu. "The experience of ethics: Evaluation of self-harm risks in automated vehicles". IEEE Intelligent Vehicles Symposium (IV). Redondo-beach, CA, USA. June 11-14, 2017.

[42] J. Pelegrín-Borondo, M. Arias-Oliva, K. Murata and M. Souto-Romero. "Does Ethical Judgment Determine the Decision to Become a Cyborg?". Journal of Business Ethics. Vol. $1 \mathrm{~N}^{\circ}$ 13. 2018. 